\title{
On the Problem of Periodic Evolution Inclusions of the Subdifferential Type
}

\author{
R. Bader and N. S. Papageorgiou
}

\begin{abstract}
We examine nonlinear periodic evolution inclusions of the subdifferential type and prove two existence theorems: one for the "non-convex, lower semicontinuous" problem and the other for the "convex, $h$-upper semicontinuous" problem. Our method of proof is based on the theory of nonlinear operators of monotone type and on multi-valued analysis. We also present three examples from partial and ordinary differential inclusions, illustrating the applicability of our work.

Keywords: Convex subdifferential, maximal monotone operators, pseudomonotone operators, operators of type $(S)_{+}$, resolvent, Yosida approximation, variational inequalities
\end{abstract}

AMS subject classification: $34 \mathrm{~K} 30,35 \mathrm{~K} 85$

\section{Introduction}

The periodic problem for differential inclusions has been studied primarily under the assumption that the orientor field (multi-valued vector field) is convex-valued. We refer to the works of Macki, Nistri and Zecca [16], Haddad and Lasry [8], Pruszko $[19]$ and the references therein. These papers deal with differential inclusions in $\mathbb{R}^{\mathbb{N}}$. The non-convex periodic problem in $\mathbb{R}^{N}$ has been considered recently by De Blasi, Gorniewicz and Pianigiani [6], $\mathrm{Hu}$, Kandilakis and Papageorgiou [10] and by $\mathrm{Hu}$ and Papageorgiou [11].

The study of the periodic problem for evolution inclusions is lagging behind. Only the "convex" problem has been investigated using a Nagumo-type tangential condition. Bader [2] considered semilinear problems and used semigroup theory and the Hausdorff measure of non-compactness. Hu and Papageorgiou [12] considered nonlinear problems driven by time-varying maximal monotone coercive operators defined in the context of an evolution triple and used Garlekin approximations. The work of Bader [2] extended to evolution inclusions the paper of Prüss [18], while the work of $\mathrm{Hu}$ and Papageorgiou [12] is related to the papers of Vrabie [21] and Hirano [9]. We should also mention the recent work of Avgerinos and Papageorgiou [1],

R. Bader: Zentrum Math. der Techn. Univ., Arcisstrasse 21, D-80333 München N. S. Papageorgiou: Nat. Techn. Univ., Dept. Math., Zografou Campus, Athens 15780, Greece bader@appl-math.tu-muenchen.de and npapg@math.ntua.gr 
who considered evolution equations defined in the framework of an evolution triple and driven by a time-varying pseudomonotone (in general not maximal monotone) operator.

In this paper we examine both the "convex" and the "non-convex" periodic problem for nonlinear evolution inclusions of the subdifferential type. Our work here appears to be the first on nonlinear, non-convex periodic evolution inclusions and also extends to a multi-valued setting the work of Hirano [9]. Our approach is based on techniques from the theory of nonlinear operators of monotone type and from multi-valued analysis.

\section{Mathematical background}

For easy reference, in this section we present some basic definitions and facts from nonlinear operator theory and multi-valued analysis, which we shall need in the sequel. Our main sources are the books $[13,14,22]$.

Let $(\Omega, \Sigma)$ be a measurable space and $X$ a separable Banach space. Throughout this paper we use the notations

$$
\begin{aligned}
P_{f(c)}(X) & =\{A \subseteq X: A \text { is non-empty, closed (and convex) }\} \\
P_{(w) k(c)}(X) & =\{A \subseteq X: A \text { is non-empty, (weakly-) compact (and convex) }\} .
\end{aligned}
$$

A multifunction (set-valued function) $F: \Omega \rightarrow P_{f}(X)$ is said to be measurable, if for each $x \in X$ the function

$$
\omega \mapsto d(x, F(\omega))=\inf \{\|x-u\|: u \in F(\omega)\}
$$

is $\Sigma$-measurable. Also, the multifunction $F: \Omega \rightarrow 2^{X} \backslash\{\emptyset\}$ is said to be graph measurable, if

$$
\operatorname{Gr} F=\{(\omega, x) \in \Omega \times X: x \in F(\omega)\} \in \Sigma \times B(X)
$$

with $B(X)$ being the Borel $\sigma$-field of $X$. For a multifunction with values in $P_{f}(X)$, measurability implies graph measurability, while the converse is true if $\Sigma$ is complete (i.e. $\Sigma=\hat{\Sigma}=$ the universal $\sigma$-field).

Now let $\mu$ be a finite measure on $\Sigma$. Given a multifunction $F: \Omega \rightarrow 2^{X} \backslash\{\emptyset\}$ and $1 \leq p \leq \infty$, we define the set

$$
S_{F}^{p}=\left\{f \in L^{p}(\Omega, X): f(\omega) \in F(\omega) \mu \text {-a.e. on } \Omega\right\}
$$

which may be empty. An easy application of a measurable selection theorem shows that, for a graph measurable function $F$, the set $S_{F}^{p}$ is non-empty if and only if

$$
\inf \{\|u\|: u \in F(\omega)\} \leq \varphi(\omega) \quad \mu \text {-a.e. on } \Omega
$$


with $\varphi \in L^{p}(\Omega)_{+}$. Moreover, the set $S_{F}^{p}$ is closed or convex if and only if for $\mu$-almost all $\omega \in \Omega$ the set $F(\omega)$ is closed or convex, respectively. Also, if $F: \Omega \rightarrow P_{w k c}(X)$ is measurable and

$$
|F(\omega)|=\sup \{\|u\|: u \in F(\omega)\} \leq \varphi_{1}(\omega) \mu \text {-a.e. on } \Omega
$$

with $\varphi_{1} \in L^{p}(\Omega)_{+}(1 \leq p<\infty)$, then $S_{F}^{p} \subset L^{p}(\Omega, X)$ is non-empty, weakly compact and convex. The set $S_{F}^{\bar{p}}$ is decomposable in the sense that if $\left(A, f_{1}, f_{2}\right) \in \Sigma \times S_{F}^{p} \times S_{F}^{p}$, then $\chi_{A} f_{1}+\chi_{A^{\text {c }}} f_{2} \in S_{F}^{p}$.

Let $Y$ and $Z$ be Hausdorff topological spaces. A multifunction $G: Y \rightarrow 2^{Z}$ is said to be lower semicontinuous, if for every $C \subset Z$ closed, the set

$$
G^{+}(C)=\{y \in Y: G(y) \subset C\}
$$

is closed. If $Z$ is a metric space with metric $d$, then the multifunction $G$ is lower semicontinuous if and only if for any $z \in Z$ the function $y \rightarrow d(z, G(y))$ is upper semicontinuous. Also, if $Z$ is a metric space with metric $d$ on $P_{f}(Z)$ we can define a generalized metric, known in the literature as Hausdorff metric, by setting

$$
\left.h(A, B)=\max \sup _{a \in A} d(a, B), \sup _{b \in B} d(b, A)\right] \quad\left(A, B \in P_{f}(Z)\right) .
$$

If $Z$ is a complete metric space, then so is $\left(P_{f}(Z), h\right)$. A multifunction $G: Y \rightarrow P_{f}(Z)$ is said to be $h$-continuous, if it is continuous from $Y$ into the metric space $\left(P_{f}(Z), h\right)$. Also, we set

$$
h^{*}(A, B)=\sup _{a \in A} d(a, B)
$$

and a multifunction $G: Y \rightarrow 2^{Z} \backslash\{\emptyset\}$ is said to be h-upper semicontinuous if for all $y \in Y$ the function $v \rightarrow h^{*}(G(v), G(y))$ is continuous at $y \in Y$.

Next, let $X$ be a reflexive Banach space and $X^{*}$ its (topological) dual. A map $A: D \subset X \rightarrow 2^{X^{*}}$ is said to be monotone, if $\left\langle x^{*}-y^{*}, x-y\right\rangle \geq 0$ for all $x, y \in D$ and all $x^{*} \in A(x), y^{*} \in A(y)$. Here by $\langle\cdot, \cdot\rangle$ we denote the duality brackets for the pair $\left(X, X^{*}\right)$. If in addition the equality $\left\langle x^{*}-y^{*}, x-y\right\rangle=0$ implies $x=y$, then $A$ is strictly monotone. The map $A$ is said to be maximal monotone if $\left\langle x^{*}-y^{*}, x-y\right\rangle \geq 0$ for all $x \in D$ and all $x^{*} \in A(x)$ imply that $y \in D$ and $y^{*} \in A(y)$, i.e. the graph of $A$ is maximal with respect to inclusion among the graphs of all monotone maps. It is easy to see that the graph of a maximal monotone map is closed in $X \times X_{w}^{*}$ and in $X_{w} \times X^{*}$. Here by $X_{w}$ and $X_{w}^{*}$ we denote the spaces $X$ and $X^{*}$, respectively, furnished with the weak topology. If $X=H$ is a Hilbert space and $H^{*}=H$ (pivot space), for every maximal monotone operator $A: D \subset H \rightarrow 2^{H}$ and every $\lambda>0$, we define the two well-known operators

$$
\begin{array}{ll}
J_{\lambda}=(I+\lambda A)^{-1} & \\
A_{\lambda}=\frac{1}{\lambda}\left(I-J_{\lambda}\right) & \text { (the resolvent of } A) \\
\text { (the Yosida approximation of } A) .
\end{array}
$$

We have $D\left(J_{\lambda}\right)=D\left(A_{\lambda}\right)=H$ for all $\lambda>0$. Both operators $J_{\lambda}$ and $A_{\lambda}$ are singlevalued and have nice properties which are listed below: 
(a) $J_{\lambda}$ is non-expansive, i.e. $\left\|J_{\lambda}(x)-J_{\lambda}(y)\right\| \leq\|x-y\|$ for all $x, y \in H$.

(b) $A_{\lambda}$ is monotone and Lipschitz continuous with Lipschitz constant $\frac{1}{\lambda}$ (hence $A_{\lambda}$ is maximal monotone).

(c) $A_{\lambda}(x) \in A\left(J_{\lambda}(x)\right)$ for all $x \in H$.

(d) $\left\|A_{\lambda}(x)\right\| \leq\left\|A^{0}(x)\right\|$ for all $x \in D$, where $A^{0}(x)$ is the unique element of minimal norm in $A(x)$ and $A_{\lambda}(x) \rightarrow A^{0}(x)$ as $\lambda \downarrow 0$ for all $x \in D$.

(e) $\bar{D}$ is convex and $J_{\lambda}(x) \rightarrow \operatorname{proj}(x ; \bar{D})$ for all $x \in H$ where $\operatorname{proj}(\cdot ; \bar{D})$ denotes the metric projection on the convex set $\bar{D}$.

Let $\varphi: H \rightarrow \overline{\mathbb{R}}=\mathbb{R} \cup\{+\infty\}$ be a proper (i.e. the set $\{x \in H: \varphi(x)<+\infty\}$ is non-empty), convex and lower semicontinuous (i.e. $\left.\varphi \in \Gamma_{0}(H)\right)$ function. The effective domain of $\varphi$ is the set

$$
\operatorname{dom} \varphi=\{x \in H: \varphi(x)<+\infty\} .
$$

The subdifferential of $\varphi$ is the multi-valued operator $\partial \varphi: D(\partial \varphi) \subset H \rightarrow 2^{H}$ defined by

$$
\partial \varphi(x)=\{\in H:\langle u, y-x\rangle \leq \varphi(y)-\varphi(x) \text { for all } y \in H\} .
$$

We have $D(\partial \varphi) \subset \operatorname{dom} \varphi$ and $\partial \varphi$ is a maximal monotone operator. For $\lambda>0$ we define

$$
\varphi_{\lambda}(x)=\inf \left[\varphi(y)+\frac{1}{2 \lambda}\|x-y\|^{2}: y \in Y\right]
$$

and call $\varphi_{\lambda}$ the Moreau-Yosida approximation of $\varphi$. We know that

- $\varphi_{\lambda}$ is convex and Fréchet differentiable (hence continuous)

- $\varphi_{\lambda}^{\prime}(x)=\partial \varphi_{\lambda}(x)=(\partial \varphi)_{\lambda}(x)$

- $\varphi\left(J_{\lambda}(x)\right) \leq \varphi_{\lambda}(x) \leq \varphi(x)$ for all $\lambda>0$ and all $x \in H$

- $\varphi_{\lambda}(x) \rightarrow \varphi(x)$ as $\lambda \downarrow 0$ for all $x \in H$.

Now return to the more general situation where $X$ is a reflexive Banach space. A single-valued and everywhere defined operator $A: X \rightarrow X^{*}$ is said to be demicontinuous if $x_{n} \rightarrow x$ in $X$ implies $A\left(x_{n}\right) \stackrel{w}{\rightarrow} A(x)$ in $X^{*}$. A monotone demicontinuous operator is maximal monotone. A map $A: D \subset X \rightarrow 2^{X^{*}}$ is said to be coercive if inf $\left[\left\|x^{*}\right\|_{*}: x^{*} \in A(x)\right] \rightarrow \infty$ as $\|x\| \rightarrow \infty$ where $\|\cdot\|$ denotes the norm of $X$ and $\|\cdot\|_{*}$ the norm of $X^{*}$. A maximal monotone coercive operator is surjective.

An operator $A: X \rightarrow X^{*}$ is said to be pseudomonotone, if

$$
\left.\begin{array}{l}
x_{n} \stackrel{w}{\rightarrow} x \text { in } X \\
A\left(x_{n}\right) \stackrel{w}{\rightarrow} u \text { in } X^{*} \\
\limsup \left\langle A\left(x_{n}\right), x_{n}-x\right\rangle \leq 0
\end{array}\right\} \Longrightarrow\left\{\begin{array}{l}
u=A(x) \\
\left\langle A\left(x_{n}\right), x_{n}\right\rangle \rightarrow\langle A(x), x\rangle .
\end{array}\right.
$$

A monotone demicontinuous map is pseudomonotone. The sum of pseudomonotone maps is still pseudomonotone. Also, a pseudomonotone coercive map is surjective. Finally, a map $A: X \rightarrow X^{*}$ is said to be of type $(S)_{+}$if

$$
\left.\begin{array}{l}
x_{n} \stackrel{w}{\rightarrow} x \\
\limsup \left\langle A\left(x_{n}\right), x_{n}-x\right\rangle \leq 0
\end{array}\right\} \quad \Longrightarrow \quad x_{n} \rightarrow x \text { in } X .
$$


The prototype map of type $(S)_{+}$is a uniformly monotone map $A: X \rightarrow X^{*}$, i.e. $A$ satisfies

$$
\psi(\|x-y\|)\|x-y\| \leq\langle A(x)-A(y), x-y\rangle \quad(x, y \in X)
$$

where $\psi: \mathbb{R}_{+} \rightarrow \mathbb{R}_{+}$a strictly monotone, increasing and continuous with $\psi(0)=0$ and $\lim _{r \rightarrow \infty} \psi(r)=+\infty$. A demicontinuous map of type $(S)_{+}$is pseudomonotone.

\section{Non-convex problem}

Let $T=[0, b]$ and $H$ a separable Hilbert space with inner product $(\cdot, \cdot)$. We study the multi-valued periodic problem

$$
\left.\begin{array}{ll}
-\dot{x}(t) \in \partial \varphi(x(t))+F(t, x(t)) & \text { a.e. on } T \\
x(0)=x(b) &
\end{array}\right\}
$$

where $\varphi \in \Gamma_{0}(H)$ and $F: T \times H \rightarrow 2^{H} \backslash\{\emptyset\}$. The precise hypotheses on the data of this problem are the following ones:

$\mathbf{H}(\varphi) \varphi \in \Gamma_{0}(H)$ is of compact-type, i.e. the set $\left\{x \in H: \varphi(x)+\|x\|^{2} \leq \theta\right\}$ is compact for all $\theta \geq 0$ and $0 \in \partial \varphi(0)$.

Remark. We know that $\varphi$ is of compact type if and only if for every $\lambda>0$ the resolvent $J_{\lambda}$ of $\partial \varphi$ is compact (see [13: p. 412]). Also, the condition $0 \in \partial \varphi(0)$ implies that $\varphi(0)=\inf _{H} \varphi$, i.e. $\varphi$ attains its infimum at $x=0$.

$\mathbf{H}(\mathbf{F})_{1} F: T \times H \rightarrow P_{f}(H)$ is a multifunction such that the following conditions are satisfied:

(i) $(t, x) \rightarrow F(t, x)$ is graph measurable.

(ii) For a.a. $t \in T, x \rightarrow F(t, x)$ is lower semicontinuous.

(iii) For a.a. $t \in T$, all $x \in H$ and all $v \in F(t, x),\|v\| \leq c_{1}(t)+c_{2}(t)\|x\|$ with $c_{1}, c_{2} \in L^{2}(T)_{+}$.

(iv) For a.a. $t \in T$, all $x \in D(\partial \varphi)$, all $w \in \partial \varphi(x)$ and all $v \in F(t, x),(w+v, x) \geq$ $c_{3}\|x\|^{2}-c_{4}(t)$ with $c_{3}>0$ and $c_{4} \in L^{1}(T)_{+}$.

Definition. A function $x \in W^{1,2}(T, H)$ is said to be a strong solution of problem (1) if $x(t) \in D(\partial \varphi)$ for all $t \in T, x(0)=x(b)$ and there exist $u \in S_{\partial \varphi(x(\cdot))}^{2}$ and $f \in S_{F(\cdot, x(\cdot))}^{2}$ such that $-\dot{x}(t)=u(t)+f(t)$ a.e. on $T$.

Remark. We know (see, for example, [14: p. 6]) that a function $x \in W^{\mathbf{1 , 2}}(T, H)$ is absolutely continuous, hence strongly differentiable almost everywhere on $T$.

Consider the vectorial Sobolev space $W_{p e r}^{1,2}(T, H)$ defined by

$$
W_{\text {per }}^{1,2}=\left\{x \in W^{1,2}(T, H): x(0)=x(b)\right\} .
$$

Since $W^{1,2}(T, H) \subset C(T, H)$, the pointwise evaluations at $t=0$ and $t=b$ make sense. Let $W_{\text {per }}^{1,2}(T, H)^{*}$ be the dual of $W_{\text {per }}^{1,2}(T, H)$. Then the triple

$$
\left(W_{p e r}^{1,2}(T, H), L^{2}(T, H), W_{p e r}^{1,2}(T, H)^{*}\right)
$$


is an evolution triple (see [14: p. 3]) or [22: p. 416]) and by $\langle\cdot, \cdot\rangle$ we denote the duality brackets for the pair $\left(W_{p e r}^{1,2}(T, H), W_{\text {per }}^{1,2}(T, H)^{*}\right)$. Note that $\left.\langle\cdot, \cdot\rangle\right|_{W_{p e r}^{1,2}(T, H) \times L^{2}(T, H)}$ is the inner product in the Hilbert space $L^{2}(T, H)$. Also, let $\hat{J}_{\frac{1}{n}}: L^{2}(T, H) \rightarrow L^{2}(T, H)$ be the Nemitsky operator corresponding to the resolvent operator $J_{\frac{1}{n}}$ of the maximal monotone map $\partial \varphi$, i.e. $\hat{J}_{\frac{1}{n}}(x)(\cdot)=J_{\frac{1}{n}}(x(\cdot))$ for all $x \in L^{2}(T, H)$.

Let

$$
R: C(T, H) \rightarrow P_{f}\left(L^{2}(T, H)\right)
$$

be the multi-valued Nemitsky operator corresponding to $F$, i.e. $R(x)=S_{F(\cdot, x(\cdot))}^{2}$.

Proposition 1. If hypotheses $H(F)_{1}$ hold, then $R$ is lower semicontinuous.

Proof. By what was said in Section 2, it suffices to show that for every $v \in$ $L^{2}(T, H)$ the function $x \rightarrow d(v, R(x))$ is upper semicontinuous from $C(T, H)$ into $\mathbb{R}_{+}$. To this end we have to prove that for every $\theta>0$ the superlevel set

$$
U(\theta)=\{x \in C(T, H): d(v, R(x)) \geq \theta\}
$$

is closed. Let $x_{n} \in U(\theta)(n \geq 1)$ and assume that $x_{n} \rightarrow x$ in $C(T, H)$. Then by Fatou's Lemma (hypothesis $H(F)_{1} /(i i i)$ permits its use) we have

$$
\limsup _{n \rightarrow \infty} \int_{0}^{b} d\left(v(t), F\left(t, x_{n}(t)\right)\right) d t \leq \int_{0}^{b} \limsup _{n \rightarrow \infty} d\left(v(t), F\left(t, x_{n}(t)\right)\right) d t .
$$

Because $F(t, \cdot)$ is lower semicontinuous for almost all $t \in T, y \rightarrow d(v(t), F(t, y))$ is upper semicontinuous. So since $x_{n}(t) \rightarrow x(t)$ for all $t \in T$ we have

$$
\limsup _{n \rightarrow \infty} d\left(v(t), F\left(t, x_{n}(t)\right)\right) \leq d(v(t), F(t, x(t)))
$$

a.e. on $T$. Hence

$$
\limsup _{n \rightarrow \infty} \int_{0}^{b} d\left(v(t), F\left(t, x_{n}(t)\right)\right) d t \leq \int_{0}^{b} d(v(t), F(t, x(t))) d t .
$$

But we know that

$$
\begin{aligned}
\int_{0}^{b} d\left(v(t), F\left(t, x_{n}(t)\right)\right) d t & =d\left(v, R\left(x_{n}\right)\right) \quad(n \geq 1) \\
\int_{0}^{b} d(v(t), F(t, x(t))) d t & =d(v, R(x))
\end{aligned}
$$

(see [13: p. 183]). Therefore $\theta \leq d(v, R(x))$ and we have proved the closedness of $U(\theta)$. So $R$ is lower semicontinuous as claimed by the proposition 
Note that the values of $R$ are decomposable subsets of $L^{2}(T, H)$. So we can apply [13: p. 245/Theorem II.8.7] and obtain a continuous function $u: C(T, H) \rightarrow$ $L^{2}(T, H)$ such that $u(x) \in R(x)$ for all $x \in C(T, H)$.

Now let

$$
K_{n}: W_{\text {per }}^{1,2}(T, H) \rightarrow W_{\text {per }}^{1,2}(T, H)^{*}
$$

be defined by

$$
\left\langle K_{n}(x), y\right\rangle=\left(\left(\frac{1}{n} x^{\prime}, y^{\prime}\right)\right)+\left(\left(\frac{1}{n} x, y\right)\right)+\left(\left(x^{\prime}+u\left(\hat{J}_{\frac{1}{n}}(x)\right), y\right)\right)
$$

for all $x, y \in W_{\text {per }}^{1,2}(T, H)$. Here by $((\cdot, \cdot))$ we denote the inner product for the Hilbert space $L^{2}(T, H)$, i.e. $((f, g))=\int_{0}^{b}(f(t), g(t)) d t$. Also, note that since $W_{\text {per }}^{1,2}(T, H) \subset$ $C(T, H)$, from the properties of the resolvent operator (see Section 2) we have $\hat{J}_{\frac{1}{n}}(x)(\cdot)=J_{\frac{1}{n}}(x(\cdot)) \in C(T, H)$ for all $x \in W_{\text {per }}^{1,2}(T, H)$ and so $u\left(\hat{J}_{\frac{1}{n}}(x)\right)$ is well defined.

Proposition 2. If hypotheses $H(\varphi)$ and $H(F)_{1}$ hold, then $K_{n}$ is demicontinuous and of type $(S)_{+}$.

Proof. First we show the demicontinuity of $K_{n}$. To this end let $x_{m} \rightarrow x$ in $W_{\text {per }}^{1,2}(T, H)$. Then $x_{m}^{\prime} \rightarrow x^{\prime}$ in $L^{2}(T, H)$ and from the non-expansiveness of $J_{\frac{1}{n}}(\cdot)$ we have

$$
\left\|J_{\frac{1}{n}}\left(x_{m}(t)\right)-J_{\frac{1}{n}}(x(t))\right\| \leq\left\|x_{m}(t)-x(t)\right\|
$$

for all $t \in T$ and $m \geq 1$ from which

$$
\left\|\hat{J}_{\frac{1}{n}}\left(x_{m}\right)-\hat{J}_{\frac{1}{n}}(x)\right\|_{C(T, H)} \leq\left\|x_{m}-x\right\|_{C(T, H)}
$$

follows. But because $x_{m} \rightarrow x$ in $W_{p e r}^{1,2}(T, H)$ and $W_{p e r}^{1,2}(T, H)$ is embedded continuously in $C(T, H),\left\|x_{m}-x\right\|_{C(T, H)} \rightarrow 0$ as $m \rightarrow \infty$, hence $\hat{J}_{\frac{1}{n}}\left(x_{m}\right) \rightarrow \hat{J}_{\frac{1}{n}}(x)$ in $C(T, H)$ as $m \rightarrow \infty$. Therefore $u\left(\hat{J}_{\frac{1}{n}}\left(x_{m}\right)\right) \rightarrow u\left(\hat{J}_{\frac{1}{n}}(x)\right)$ in $L^{2}(T, H)$ as $m \rightarrow \infty$. For every $y \in W_{p e r}^{1,2}(T, H)$ we have

$$
\left.\begin{array}{rl}
\left(\left(\frac{1}{n} x_{m}^{\prime}, y\right)\right) & \rightarrow\left(\left(\frac{1}{n} x^{\prime}, y\right)\right) \\
\left(\left(\frac{1}{n} x_{m}, y\right)\right) & \rightarrow\left(\left(\frac{1}{n} x, y\right)\right) \\
\left(\left(x_{m}^{\prime}+u\left(\hat{J}_{\frac{1}{n}}\left(x_{m}\right)\right), y\right)\right) & \rightarrow\left(\left(x^{\prime}+u\left(\hat{J}_{\frac{1}{n}}(x)\right), y\right)\right)
\end{array}\right\} \quad(m \rightarrow \infty) .
$$

It follows that $\left\langle K_{n}\left(x_{m}\right), y\right\rangle \rightarrow\left\langle K_{n}(x), y\right\rangle$ as $m \rightarrow \infty$, which proves the demicontinuity of $K_{n}$.

Next we show that $K_{n}$ is of type $(S)_{+}$. So suppose that $x_{m} \stackrel{w}{\rightarrow} x$ in $W_{p e r}^{1,2}(T, H)$ and assume $\lim \sup _{m \rightarrow \infty}\left\langle K_{n}\left(x_{m}\right), x_{m}-x\right\rangle \leq 0$. We have to show that $x_{m} \rightarrow x$ in $W_{p e r}^{1,2}(T, H)$ as $m \rightarrow \infty$. From the definition of the operator $K_{n}$,

$$
\begin{aligned}
\left\langle K_{n}\left(x_{m}\right), x_{m}-x\right\rangle= & \left(\left(\frac{1}{n} x_{m}^{\prime}, x_{m}^{\prime}-x^{\prime}\right)\right)+\left(\left(\frac{1}{n} x_{m}, x_{m}-x\right)\right) \\
& +\left(\left(x_{m}^{\prime}+u\left(\hat{J}_{\frac{1}{n}}\left(x_{m}\right)\right), x_{m}-x\right)\right) .
\end{aligned}
$$


Because $x_{m} \stackrel{w}{\rightarrow} x$ in $W_{p e r}^{1,2}(T, H), x_{m} \stackrel{w}{\rightarrow} x$ in $C(T, H)$ and so $x_{m}(t) \stackrel{w}{\rightarrow} x(t)$ in $H$ as $m \rightarrow \infty$, for all $t \in T$. Since $\varphi$ is of compact type (hypothesis $H(\varphi)), J_{\frac{1}{n}}(\cdot)$ is a compact operator (see [13: p. 412]) and so $J_{\frac{1}{n}}\left(x_{m}(t)\right) \rightarrow J_{\frac{1}{n}}(x(t))$ in $H$ as $m \rightarrow \infty$, for all $t \in T$. Thus, for each $t \in T,\left\{J_{\frac{1}{n^{n}}}\left(x_{m}(t)\right)\right\}_{m \geq 1}$ is relatively compact. Also, we know that $x_{m}^{\prime} \stackrel{w}{\rightarrow} x^{\prime}$ in $L^{2}(T, H)$ and so $\left\{x_{m}^{\prime}\right\}_{m \geq 1}$ is uniformly integrable. Hence given $t \in T$ and $\varepsilon>0$ there exists $0<\delta=\delta(t, \varepsilon)$ such that

$$
\int_{t}^{t+\delta}\left\|x_{m}^{\prime}(s)\right\| d s<\varepsilon \quad(m \geq 1)
$$

Hence for $\hat{t} \in[t, t+\delta)$

$$
\left\|J_{\frac{1}{n}}\left(x_{m}(\hat{t})\right)-J_{\frac{1}{n}}\left(x_{m}(t)\right)\right\| \leq\left\|x_{m}(\hat{t})-x_{m}(t)\right\| \leq \int_{t}^{\hat{t}}\left\|x_{m}^{\prime}(s)\right\| d s<\varepsilon
$$

and we see that $\left\{J_{\frac{1}{n}}\left(x_{m}(\cdot)\right)\right\}_{m \geq 1}$ is also equicontinuous. By the Ascoli-Arzela theorem it follows that $\left\{\hat{J}_{\frac{1}{n}}\left(x_{m}\right)\right\}_{m \geq 1} \subset C(T, H)$ is relatively compact. Since $u$ is continuous, we obtain the same conclusion for $\left\{u\left(\hat{J}_{\frac{1}{n}}\left(x_{m}\right)\right)\right\}_{m \geq 1} \subset L^{2}(T, H)$. Moreover, because $x_{m} \stackrel{w}{\rightarrow} x$ in $W_{\text {per }}^{1,2}(T, H), x_{m} \stackrel{w}{\rightarrow} x$ in $L^{2}(T, H)$ and so

$$
\lim _{m \rightarrow \infty}\left(\left(u\left(\hat{J}_{\frac{1}{n}}\left(x_{m}\right)\right), x_{m}-x\right)\right)=0 .
$$

Also, for all $m \geq 1$

$$
\left(\left(x_{m}^{\prime}, x_{m}-x\right)\right)=\int_{0}^{b} \frac{1}{2} \frac{d}{d t}\left\|x_{m}(t)\right\|^{2} d t-\left(\left(x_{m}^{\prime}, x\right)\right)=-\left(\left(x_{m}^{\prime}, x\right)\right)
$$

since $x_{m}(0)=x_{m}(b)$ and so

$$
\begin{aligned}
\lim _{m \rightarrow \infty}\left(\left(x_{m}^{\prime}, x_{m}-x\right)\right) & =-\lim _{m \rightarrow \infty}\left(\left(x_{m}^{\prime}, x\right)\right) \\
& =-\left(\left(x^{\prime}, x\right)\right) \\
& =-\int_{0}^{b} \frac{d}{d t}\|x(t)\|^{2} d t \\
& =0 .
\end{aligned}
$$

We return to (2), pass to the limit as $m \rightarrow \infty$ and use (3) - (4) above. So

$$
\begin{aligned}
& \limsup _{m \rightarrow \infty}\left[\left(\left(\frac{1}{n} x_{m}^{\prime}, x_{m}^{\prime}-x^{\prime}\right)\right)+\left(\left(\frac{1}{n} x_{m}, x_{m}-x\right)\right)\right] \leq 0 \\
\Longrightarrow & \limsup _{m \rightarrow \infty}\left[\left(\left(\frac{1}{n}\left(x_{m}^{\prime}-x^{\prime}\right), x_{m}^{\prime}-x^{\prime}\right)\right)+\left(\left(\frac{1}{n}\left(x_{m}-x\right), x_{m}-x\right)\right)\right] \leq 0 \\
\Longrightarrow & \limsup _{m \rightarrow \infty}\left[\frac{1}{n}\left\|x_{m}^{\prime}-x^{\prime}\right\|_{2}^{2}+\frac{1}{n}\left\|x_{m}-x\right\|_{2}^{2}\right] \leq 0 \\
\Longrightarrow & \left\|x_{m}-x\right\|_{W^{1,2}(T, H)} \rightarrow 0(m \rightarrow \infty) .
\end{aligned}
$$

Therefore we have proved that $K_{n}$ is of type $(S)_{+}$ 
For $\lambda>0$ let $A_{\lambda}: H \rightarrow H$ be the Yosida approximation of the maximal monotone operator $A=\partial \varphi$. Recall that $A_{\lambda}=\partial \varphi_{\lambda}$ (see Section 2). Let

$$
\hat{A}_{\lambda}: L^{2}(T, H) \rightarrow L^{2}(T, H)
$$

be the Nemitsky operator corresponding to $A_{\lambda}$, i.e. $\hat{A}_{\lambda}(x)(\cdot)=A_{\lambda}(x(\cdot))$.

Proposition 3. If hypotheses $H(\varphi)$ and $H(F)_{1}$ hold, then for $n \geq 1$ large $K_{n}+$ $\hat{A}_{\frac{1}{n}}$ is coercive.

Proof. Suppose that $\left\|x_{m}\right\|_{W^{1,2}(T, H)} \rightarrow \infty$ as $m \rightarrow \infty$. We have

$$
\begin{aligned}
& \left\langle K_{n}\left(x_{m}\right)+\hat{A}_{\frac{1}{n}}\left(x_{m}\right), x_{m}\right\rangle \\
& \quad=\frac{1}{n}\left\|x_{m}\right\|_{W^{1,2}(T, H)}^{2}+\left(\left(x_{m}^{\prime}, x_{m}\right)\right)+\left(\left(\hat{A}_{\frac{1}{n}}\left(x_{m}\right)+u\left(\hat{J}_{\frac{1}{n}}\left(x_{m}\right)\right), x_{m}\right)\right) .
\end{aligned}
$$

From the definition of the Yosida approximation, $\hat{A}_{\frac{1}{n}}=n\left(I-\hat{J}_{\frac{1}{n}}\right)$. So

$$
\begin{aligned}
\left(\left(\hat{A}_{\frac{1}{n}}\left(x_{m}\right), x_{m}\right)\right) & =\left(\left(n\left(I-\hat{J}_{\frac{1}{n}}\right)\left(x_{m}\right), x_{m}\right)\right) \\
& =\left(\left(n\left(I-\hat{J}_{\frac{1}{n}}\right)\left(x_{m}\right),\left(I-\hat{J}_{\frac{1}{n}}\right)\left(x_{m}\right)\right)\right)+\left(\left(\hat{A}_{\frac{1}{n}}\left(x_{m}\right), \hat{J}_{\frac{1}{n}}\left(x_{m}\right)\right)\right) \\
& =n\left\|\left(I-\hat{J}_{\frac{1}{n}}\right)\left(x_{m}\right)\right\|_{2}^{2}+\left(\left(\hat{A}_{\frac{1}{n}}\left(x_{m}\right), \hat{J}_{\frac{1}{n}}\left(x_{m}\right)\right)\right) .
\end{aligned}
$$

Also,

$$
\left(\left(x_{m}^{\prime}, x_{m}\right)\right)=\frac{1}{2} \int_{0}^{b} \frac{d}{d t}\left\|x_{m}(t)\right\|^{2} d t=0
$$

because $x_{m}(0)=x_{m}(b)$. Therefore returning to (5) we can write

$$
\begin{aligned}
\left\langle K_{n}\left(x_{m}\right)+\hat{A}_{\frac{1}{n}}\left(x_{m}\right), x_{m}\right\rangle= & \frac{1}{n}\left\|x_{m}\right\|_{W^{1,2}(T, H)}^{2}+n\left\|\left(I-\hat{J}_{\frac{1}{n}}\right)\left(x_{m}\right)\right\|_{2}^{2} \\
& +\left(\left(\hat{A}_{\frac{1}{n}}\left(x_{m}\right)+u\left(\hat{J}_{\frac{1}{n}}\left(x_{m}\right)\right), \hat{J}_{\frac{1}{n}}\left(x_{m}\right)\right)\right) \\
& +\left(\left(u\left(\hat{J}_{\frac{1}{n}}\left(x_{m}\right)\right), x_{m}-\hat{J}_{\frac{1}{n}}\left(x_{m}\right)\right)\right) .
\end{aligned}
$$

We know that

$$
\begin{aligned}
& A_{\frac{1}{n}}\left(x_{m}(t)\right) \in A\left(J_{\frac{1}{n}}\left(x_{m}(t)\right)\right) \quad(t \in T) \\
& u\left(\hat{J}_{\frac{1}{n}}\left(x_{m}\right)\right)(t) \in F\left(t, J_{\frac{1}{n}}\left(x_{m}(t)\right)\right) \text { a.e. on } T \quad(m \geq 1) \text {. }
\end{aligned}
$$

Using hypothesis $H(F)_{1} /(i v)$, we get

$$
\left(\left(\hat{A}_{\frac{1}{n}}\left(x_{m}\right)+u\left(\hat{J}_{\frac{1}{n}}\left(x_{m}\right)\right), \hat{J}_{\frac{1}{n}}\left(x_{m}\right)\right)\right) \geq c_{5}\left\|\hat{J}_{\frac{1}{n}}\left(x_{m}\right)\right\|_{2}^{2}-c_{6}
$$

for $m \geq 1$ with $c_{5}, c_{6}>0$. Also, from hypothesis $H(F)_{1} /(i i i)$ we have

$$
\left|\left(\left(u\left(\hat{J}_{\frac{1}{n}}\left(x_{m}\right)\right), x_{m}-\hat{J}_{\frac{1}{n}}\left(x_{m}\right)\right)\right)\right| \leq\left(c_{7}+c_{8}\left\|\hat{J}_{\frac{1}{n}}\left(x_{m}\right)\right\|_{2}\right)\left\|x_{m}-\hat{J}_{\frac{1}{n}}\left(x_{m}\right)\right\|_{2}
$$


for $m \geq 1$ with $c_{7}, c_{8}>0$. Using Young's inequality with $\varepsilon>0$ on the right-hand side we obtain

$$
\begin{aligned}
& \left(c_{7}+c_{8}\left\|\hat{J}_{\frac{1}{n}}\left(x_{m}\right)\right\|_{2}\right)\left\|x_{m}-\hat{J}_{\frac{1}{n}}\left(x_{m}\right)\right\|_{2} \\
& \quad \leq \varepsilon c_{6}^{2}+\varepsilon c_{7}^{2}\left\|\hat{J}_{\frac{1}{n}}\left(x_{m}\right)\right\|_{2}^{2}+\frac{1}{2 \varepsilon}\left\|x_{m}-\hat{J}_{\frac{1}{n}}\left(x_{m}\right)\right\|_{2}^{2}
\end{aligned}
$$

from which

$$
\begin{aligned}
& \left|\left(\left(u\left(\hat{J}_{\frac{1}{n}}\left(x_{m}\right)\right), x_{m}-\hat{J}_{\frac{1}{n}}\left(x_{m}\right)\right)\right)\right| \\
& \quad \leq \varepsilon c_{6}^{2}+\varepsilon c_{7}^{2}\left\|\hat{J}_{\frac{1}{n}}\left(x_{m}\right)\right\|_{2}^{2}+\frac{1}{2 \varepsilon}\left\|x_{m}-\hat{J}_{\frac{1}{n}}\left(x_{m}\right)\right\|_{2}^{2}
\end{aligned}
$$

follows. Using (7) and (8) in (6), we obtain

$$
\begin{aligned}
& \left\langle K_{n}\left(x_{m}\right)+\hat{A}_{\frac{1}{n}}\left(x_{m}\right), x_{m}\right\rangle \\
& \quad \geq \frac{1}{n}\left\|x_{m}\right\|_{W^{1,2}(T, H)}^{2}+n\left\|\left(I-\hat{J}_{\frac{1}{n}}\right)\left(x_{m}\right)\right\|_{2}^{2} \\
& \quad+c_{5}\left\|\hat{J}_{\frac{1}{n}}\left(x_{m}\right)\right\|_{2}^{2}-\varepsilon c_{7}^{2}\left\|\hat{J}_{\frac{1}{n}}\left(x_{m}\right)\right\|_{2}^{2}-\frac{1}{2 \varepsilon}\left\|x_{m}-\hat{J}_{\frac{1}{n}}\left(x_{m}\right)\right\|_{2}^{2}-c_{8}
\end{aligned}
$$

with $c_{8}=c_{8}(\varepsilon)>0$. Choose $\varepsilon>0$ so that $c_{5}>\varepsilon c_{7}^{2}$. Then based on this choice of $\varepsilon>0$ choose $n_{0} \geq 1$ large enough so that for $n \geq n_{0}$ we have $n \geq \frac{1}{2 \varepsilon}$. With these choices, we see from (9) that for $n \geq n_{0}$ the operator $K_{n}+\hat{A}_{\frac{1}{n}}$ is coercive

Using these auxiliary results we can now prove an existence theorem for problem (1).

Theorem 4. If hypotheses $H(\varphi)$ and $H(F)_{1}$ hold, then problem (1) has a strong solution $x \in W_{\text {per }}^{1,2}\left(T, \mathbb{R}^{\mathbb{N}}\right)$.

Proof. The operator $\hat{A}_{\frac{1}{n}}$ is maximal monotone and continuous, hence pseudomonotone. Also, from Proposition 2 we know that $K_{n}$ is demicontinuous and of type $(S)_{+}$, thus pseudomonotone. The sum of pseudomonotone operators is pseudomonotone. Therefore $x \rightarrow\left(K_{n}+\hat{A}_{\frac{1}{n}}\right)(x)$ is pseudomonotone. From Proposition 3 we know that it is also coercive. Hence it is surjective (see Section 2). So for every $n \geq 1$ we can find $x_{n} \in W_{p e r}^{1,2}(T, H)$ such that

$$
K_{n}\left(x_{n}\right)+\hat{A}_{\frac{1}{n}}\left(x_{n}\right)=0
$$

From (9) and the choices of $\varepsilon>0$ and $n \geq 1$ made there (see the proof of Proposition $3)$, we have

$$
\frac{1}{n}\left\|x_{n}\right\|_{W_{\text {per }}^{1,2}(T, H)} \leq M_{1} \quad \text { for some } M_{1}>0 \text { and all } n \geq n_{0} .
$$

Also, as before, from the definition of the Yosida approximation, we have

$$
\begin{aligned}
& \left(\left(\hat{A}_{\frac{1}{n}}\left(x_{n}\right)+u\left(\hat{J}_{\frac{1}{n}}\left(x_{n}\right)\right), x_{n}\right)\right) \\
& \quad \geq c_{3}\left\|\hat{J}_{\frac{1}{n}}\left(x_{n}\right)\right\|_{2}^{2}+n\left\|\left(I-\hat{J}_{\frac{1}{n}}\right)\left(x_{n}\right)\right\|_{2}^{2} \\
& \quad-\left(c_{7}+c_{8}\left\|\hat{J}_{\frac{1}{n}}\left(x_{n}\right)\right\|_{2}\right)\left\|x_{n}-\hat{J}_{\frac{1}{n}}\left(x_{n}\right)\right\|_{2}-c_{9}
\end{aligned}
$$


for some $c_{9}>0$. Let $\beta>1$ be such that $c_{3}\left(\frac{\beta-1}{\beta}\right)^{2}>\frac{c_{8}}{\beta}$. Then

$$
\beta\left\|\left(I-\hat{J}_{\frac{1}{n}}\right)\left(x_{n}\right)\right\|_{2} \leq\left\|x_{n}\right\|_{2} \quad \Longrightarrow \quad \beta\left|\left\|x_{n}\right\|_{2}-\left\|\hat{J}_{\frac{1}{n}}\left(x_{n}\right)\right\|_{2}\right| \leq\left\|x_{n}\right\|_{2}
$$

and so

$$
(\beta-1)\left\|x_{n}\right\|_{2} \leq\left\|\hat{J}_{\frac{1}{n}}\left(x_{n}\right)\right\|_{2} .
$$

Because $0 \in \partial \varphi(0), J_{\lambda}(0)=0$ and so $\left\|\hat{J}_{\frac{1}{n}}\left(x_{n}\right)\right\|_{2} \leq\left\|x_{n}\right\|_{2}$. From (10) we obtain

$$
\begin{aligned}
& \left(\left(\hat{A}_{\frac{1}{n}}\left(x_{n}\right)+u\left(\hat{J}_{\frac{1}{n}}\left(x_{n}\right)\right), x_{n}\right)\right) \\
& \quad \geq c_{3}\left(\frac{\beta-1}{\beta}\right)^{2}\left\|x_{n}\right\|_{2}^{2}-\frac{c_{7}}{\beta}\left\|x_{n}\right\|_{2}-\frac{c_{8}}{\beta}\left\|\hat{J}_{\frac{1}{n}}\left(x_{n}\right)\right\|_{2}\left\|x_{n}\right\|_{2}-c_{9} \\
& \quad \geq c_{3}\left(\frac{\beta-1}{\beta}\right)^{2}\left\|x_{n}\right\|_{2}^{2}-\frac{c_{8}}{\beta}\left\|x_{n}\right\|_{2}^{2}-\frac{c_{7}}{\beta}\left\|x_{n}\right\|_{2}-c_{9} \\
& \quad=\left(c_{3}\left(\frac{\beta-1}{\beta}\right)^{2}-\frac{c_{8}}{\beta}\right)\left\|x_{n}\right\|_{2}^{2}-\frac{c_{7}}{\eta}\left\|x_{n}\right\|_{2}-c_{9} .
\end{aligned}
$$

On the other hand, if $\beta\left\|\left(I-\hat{J}_{\frac{1}{n}}\right)\left(x_{n}\right)\right\|_{2} \geq\left\|x_{n}\right\|_{2}$, then since $\left\|\left(I-\hat{J}_{\frac{1}{n}}\right)\left(x_{n}\right)\right\|_{2} \leq$ $2\left\|x_{n}\right\|_{2}$, from (10) we have

$$
\left(\left(\hat{A}_{\frac{1}{n}}\left(x_{n}\right)+u\left(\hat{J}_{\frac{1}{n}}\left(x_{n}\right)\right), x_{n}\right)\right) \geq\left(\frac{n}{\beta^{2}}-2 c_{8}\right)\left\|x_{n}\right\|_{2}^{2}-2 c_{7}\left\|x_{n}\right\|_{2}-c_{9}
$$

From (11) and (12) we see that, for $n \geq n_{0}$,

$$
\left(\left(\hat{A}_{\frac{1}{n}}\left(x_{n}\right)+u\left(\hat{J}_{\frac{1}{n}}\left(x_{n}\right)\right), x_{n}\right)\right) \geq c_{10}\left\|x_{n}\right\|_{2}^{2}-c_{11}
$$

for some $c_{10}, c_{11}>0$. Thus for $n \geq n_{0}$

$$
\begin{aligned}
0 & =\left\langle K_{n}\left(x_{n}\right)+\hat{A}_{\frac{1}{n}}\left(x_{n}\right), x_{n}\right\rangle \\
& \geq\left(\left(\hat{A}_{\frac{1}{n}}\left(x_{n}\right)+u\left(\hat{J}_{\frac{1}{n}}\left(x_{n}\right)\right), x_{n}\right)\right) \\
& \geq c_{10}\left\|x_{n}\right\|_{2}^{2}-c_{11} .
\end{aligned}
$$

Thus $\left\{x_{n}\right\}_{n \geq 1} \subset L^{2}(T, H)$ is bounded. From this and the fact that $\left\|\hat{J}_{\frac{1}{n}}\left(x_{n}\right)\right\|_{2} \leq$ $\left\|x_{n}\right\|_{2} \quad(n \geq 1)$ we deduce that $\left\{\hat{J}_{\frac{1}{n}}\left(x_{n}\right)\right\}_{n \geq 1} \subset L^{2}(T, H)$ is bounded. From this, the fact that $u\left(\hat{J}_{\frac{1}{n}}\left(x_{n}\right)\right) \in R\left(\hat{J}_{\frac{1}{n}}\left(x_{n}\right)\right)$ and hypothesis $H(F)_{1} /(i i i)$ it follows that

$$
\left\{u\left(\hat{J}_{\frac{1}{n}}\left(x_{n}\right)\right)\right\}_{n \geq 1} \subset L^{2}(T, H) \text { is bounded }
$$

Note that, since $x_{n} \in W_{p e r}^{1,2}(T, H)$ and $\hat{A}_{\frac{1}{n}}^{\prime}(\cdot)$ is Lipschitz continuous, $\hat{A}_{\frac{1}{n}}\left(x_{n}\right) \in$ $W^{1,2}(T, H)$. Because $K_{n}\left(x_{n}\right)+\hat{A}_{\frac{1}{n}}\left(x_{n}\right)=0(n \geq 1)$, by tacking duality brackets with $\hat{A}_{\frac{1}{n}}\left(x_{n}\right)$ we obtain

$$
0=\left\langle K_{n}\left(x_{n}\right), \hat{A}_{\frac{1}{n}}\left(x_{n}\right)\right\rangle+\left\|\hat{A}_{\frac{1}{n}}\left(x_{n}\right)\right\|_{2}^{2}
$$


and so

$$
\begin{aligned}
0= & \left(\left(\frac{1}{n} x_{n}^{\prime}, \frac{d}{d t} \hat{A}_{\frac{1}{n}}\left(x_{n}\right)\right)\right)+\left(\left(\frac{1}{n} x_{n}, \hat{A}_{\frac{1}{n}}\left(x_{n}\right)\right)\right) \\
& +\left(\left(x_{n}^{\prime}+u\left(\hat{J}_{\frac{1}{n}}\left(x_{n}\right)\right), \hat{A}_{\frac{1}{n}}\left(x_{n}\right)\right)\right)+\left\|\hat{A}_{\frac{1}{n}}\left(x_{n}\right)\right\|_{2}^{2} .
\end{aligned}
$$

Recall that $\hat{A}_{\underline{1}}$ is monotone, and because $0 \in \partial \varphi(0)$ we have $\hat{A}_{\frac{1}{n}}(0)=0$. So

$$
0 \leq\left(\left(\frac{1}{n} x_{n}, \hat{A}_{\frac{1}{n}}\left(x_{n}\right)\right)\right) \text {. }
$$

Also, for all $n \geq 1$

$$
\left(\left(\frac{1}{n} x_{n}^{\prime}, \frac{d}{d t} \hat{A}_{\frac{1}{n}}\left(x_{n}\right)\right)\right)=\int_{0}^{b}\left(\frac{1}{n} x_{n}^{\prime}(t), \frac{d}{d t} A_{\frac{1}{n}}\left(x_{n}(t)\right)\right) d t .
$$

We know that $A_{\frac{1}{n}}$ is Lipschitz continuous, and so by the generalized Rademacher theorem (see, for example, [5: p. 121]) it is Gateaux differentiable at every $x \in H \backslash D$, with $D$ being a Haar-null subset of $H$. Then, employing the chain rule of Marcus and Mizel [17],

$$
\left(x^{*}, \frac{d}{d t} A_{\frac{1}{n}}\left(x_{n}(t)\right)\right)=\frac{d}{d t}\left(x^{*}, A_{\frac{1}{n}}\left(x_{n}(t)\right)\right)=\left(x^{*}, A_{\frac{1}{n}}^{\prime}\left(x_{n}(t)\right) x_{n}^{\prime}(t)\right)
$$

for all $x^{*} \in H$ and all $t \in T \backslash N_{n}\left(x^{*}\right)$ with $\left|N_{n}\left(x^{*}\right)\right|=0$, where $|\cdot|$ is the Lebesgue measure on $T$. Let $\left\{x_{m}^{*}\right\}_{m \geq 1}$ be dense in $H$ and set $N_{n}=\cup_{m \geq 1} N_{n}\left(x_{m}^{*}\right)$. Evidently, $\left|N_{n}\right|=0$ and for $t \in T \backslash N_{n}$ and $x^{*} \in H$ we have

$$
\left(x^{*}, \frac{d}{d t} A_{\frac{1}{n}}\left(x_{n}(t)\right)\right)=\frac{d}{d t}\left(x^{*}, A_{\frac{1}{n}}\left(x_{n}(t)\right)\right)=\left(x^{*}, A_{\frac{1}{n}}^{\prime}\left(x_{n}(t)\right) x_{n}^{\prime}(t)\right)
$$

and so

$$
\frac{d}{d t} A_{\frac{1}{n}}\left(x_{n}(t)\right)=A_{\frac{1}{n}}^{\prime}\left(x_{n}(t)\right) x_{n}^{\prime}(t) \quad \text { a.e. on } T \text {. }
$$

Therefore

$$
\begin{aligned}
\left(\left(\frac{1}{n} x_{n}^{\prime}, \frac{d}{d t} \hat{A}_{\frac{1}{n}}\left(x_{n}\right)\right)\right) & =\int_{0}^{b}\left(\frac{1}{n} x_{n}^{\prime}(t), \frac{d}{d t} A_{\frac{1}{n}}\left(x_{n}(t)\right)\right) d t \\
& =\int_{0}^{b}\left(\frac{1}{n} x_{n}^{\prime}(t), A_{\frac{1}{n}}^{\prime}\left(x_{n}(t)\right) x_{n}^{\prime}(t)\right) d t .
\end{aligned}
$$

Exploiting the monotonicity of $A_{\frac{1}{n}}$ we can easily check that

$$
\left(\frac{1}{n} x_{n}^{\prime}(t), A_{\frac{1}{n}}^{\prime}\left(x_{n}(t)\right) x_{n}^{\prime}(t)\right) \geq 0 \quad \text { a.e. on } T \text {. }
$$

So we deduce

$$
0 \leq\left(\left(\frac{1}{n} x_{n}^{\prime}, \frac{d}{d t} \hat{A}_{\frac{1}{n}}\left(x_{n}\right)\right)\right) .
$$

Finally,

$$
\begin{aligned}
\left(\left(x_{n}^{\prime}, \hat{A}_{\frac{1}{n}}\left(x_{n}\right)\right)\right) & =\int_{0}^{b}\left(x_{n}^{\prime}(t), A_{\frac{1}{n}}\left(x_{n}(t)\right)\right) d t \\
& =\int_{0}^{b}\left(x_{n}^{\prime}(t), \partial \varphi_{\frac{1}{n}}\left(x_{n}(t)\right)\right) d t \\
& =\int_{0}^{b} \frac{d}{d t} \varphi_{\frac{1}{n}}\left(x_{n}(t)\right) d t \\
& =0
\end{aligned}
$$


(for the last two equalities see [13: p. 357]) and recall that $x_{n}(0)=x_{n}(b)$ ). Using (14) - (16) in (13) we obtain

$$
\left\|\hat{A}_{\frac{1}{n}}\left(x_{n}\right)\right\|_{2}^{2} \leq\left\|u\left(\hat{J}_{\frac{1}{n}}\left(x_{n}\right)\right)\right\|_{2}\left\|\hat{A}_{\frac{1}{n}}\left(x_{n}\right)\right\|_{2} .
$$

We already know that $\left\{u\left(\hat{J}_{\frac{1}{n}}\left(x_{n}\right)\right)\right\}_{n \geq 1} \subset L^{2}(T, H)$ is bounded. Therefore the sequence $\left\{\hat{A}_{\frac{1}{n}}\left(x_{n}\right)\right\}_{n \geq 1} \subset L^{2}(T, H)$ is bounded.

For every $n \geq 1, x_{n} \in\left(K_{n}+\hat{A}_{\frac{1}{n}}\right)^{-1}(0)$. From Proposition 2 we know that $K_{n}+\hat{A}_{\frac{1}{n}}$ is coercive. Therefore $\left\{x_{n}\right\}_{n \geq 1} \subset W_{\text {per }}^{1,2}(T, H)$ is bounded. By passing to a subsequence if necessary, we may assume that $x_{n} \stackrel{w}{\rightarrow} x$ in $W_{\text {per }}^{1,2}(T, H)$. Arguing as in the proof of Proposition 2, we obtain that $\left\{\hat{f}_{\frac{1}{n}}\left(x_{n}\right)\right\}_{n \geq 1} \subset L^{2}(T, H)$ is relatively compact and so we may asume that $\hat{J}_{\frac{1}{n}}\left(x_{n}\right) \rightarrow y$ in $L^{2}(T, H)$. Recall that $\hat{A}_{\frac{1}{n}}\left(x_{n}\right)=$ $n\left(I-\hat{J}_{\frac{1}{n}}\right)\left(x_{n}\right)$ and $\left\{\hat{A}_{\frac{1}{n}}\left(x_{n}\right)\right\}_{n \geq 1} \subset L^{2}(T, H)$ is bounded. So $\left\|x_{n}-\hat{J}_{\frac{1}{n}}\left(x_{n}\right)\right\|_{2} \rightarrow 0$ as $n \rightarrow \infty$, hence $x_{n} \rightarrow x=y$ in $L^{2}(T, H)$. Also, we may assume that $\hat{A}_{\frac{1}{n}}\left(x_{n}\right) \stackrel{w}{\rightarrow} v$ in $L^{2}(T, H)$.

Let $\Phi: L^{2}(T, H) \rightarrow \overline{\mathbb{R}}=\mathbb{R} \cup\{\infty\}$ be defined by

$$
\Phi(y)= \begin{cases}\int_{0}^{b} \varphi(y(t)) d t & \text { if } \varphi(y(\cdot)) \in L^{1}(T) \\ +\infty & \text { otherwise. }\end{cases}
$$

We know that $\Phi \in \Gamma_{0}\left(L^{2}(T, H)\right)$ and $\hat{A}_{\frac{1}{n}}\left(x_{n}\right)=\partial \Phi_{\frac{1}{n}}\left(x_{n}\right) \in \partial \Phi\left(\hat{J}_{\frac{1}{n}}\left(x_{n}\right)\right)$ (see [13: p 349]). The subdifferential $\partial \Phi$ ia a maximal monotone operator in the Hilbert space $L^{2}(T, H)$ and $\hat{J}_{\frac{1}{n}}\left(x_{n}\right) \rightarrow x$ in $L^{2}(T, H)$ and $\hat{A}_{\frac{1}{n}}\left(x_{n}\right) \stackrel{w}{\rightarrow} v$ in $L^{2}(T, H)$. Recalling that Gr $\partial \Phi$ is closed in $L^{2}(T, H) \times L^{2}(T, H)_{w}, v \in \partial \Phi(x)$ and so $v(t) \in \partial \varphi(x(t))$ a.e. on $T$.

Also, since $\left\{x_{n}\right\}_{n \geq 1} \subset W_{\text {per }}^{1,2}(T, H)$ is bounded, $\frac{1}{n} x_{n}, \frac{1}{n} x_{n}^{\prime} \rightarrow 0$ in $L^{2}(T, H)$. Recall that for every $z \in L^{2}(T, H)$ and all $n \geq 1$

$$
\left(\left(\frac{1}{n} x_{n}^{\prime}, z\right)\right)+\left(\left(\frac{1}{n} x_{n}, z\right)\right)+\left(\left(x_{n}^{\prime}+u\left(\hat{J}_{\frac{1}{n}}\left(x_{n}\right)\right), z\right)\right)+\left(\left(\hat{A}_{\frac{1}{n}}\left(x_{n}\right), z\right)\right)=0 .
$$

Passing to the limit as $n \rightarrow \infty$ and since $u\left(\hat{J}_{\frac{1}{n}}\left(x_{n}\right)\right) \rightarrow u(x)$ in $L^{2}(T, H)$ (because $u$ is continuous) we obtain

$$
\begin{array}{ll} 
& \left(\left(x^{\prime}+u(x), z\right)\right)+((v, z))=0 \\
\Longrightarrow \quad & x^{\prime}+u(x)+v=0 \\
\Longrightarrow \quad & -x^{\prime}(t) \in \partial \varphi(x(t))+F(t, x(t)) \text { a.e. on } T \\
& x(0)=x(b)
\end{array}
$$

because $u(x) \in R(x)$. This proves that $x \in W_{\text {per }}^{1,2}(T, H)$ is a strong solution of problem (1) 


\section{Convex problem}

In this section we prove an existence theorem for the "convex" version of problem (1). Our hypothesis on the orientor field $F(t, x)$ is the following:

$\mathrm{H}(\mathrm{F})_{2} F: T \times H \rightarrow P_{f c}(H)$ is a multifunction such that the following conditions are satisfied:

(i) $(t, x) \rightarrow F(t, x)$ is measurable.

(ii) For almost all $t \in T, x \rightarrow F(t, x)$ is $h$-upper semicontinuous.

(iii) For almost all $t \in T$, all $x \in H$ and all $v \in F(t, x),\|v\| \leq c_{1}(t)+c_{2}(t)\|x\|$ with $c_{1}, c_{2} \in L^{2}(T)_{+}$.

(iv) For almost all $t \in T$, all $x \in H$ and all $v \in F(t, x),(v, x) \geq c_{3}\|x\|^{2}-c_{4}(t)$ with $c_{3}>0$ and $c_{4} \in L^{1}(T)_{+}$.

(v) There exists $r>0$ such that, for almost all $t \in T$, all $x \in H$ with $\|x\|=r$ and all $v \in F(t, x),(v, x) \geq 0$.

Remark. If, for example, in hypothesis $H(F)_{2} /(i v)$ above $c_{4} \in L^{\infty}(T)_{+}$, then hypothesis $H(F)_{2} /(v)$ follows from hypothesis $H(F)_{2} /(i v)$.

Theorem 5. If hypotheses $H(\varphi)$ and $H(F)_{2}$ hold, then the set of strong solutions of problem (1) is non-empty and compact in $C(T, H)$.

Proof. Let $r>0$ be as in hypothesis $H(F)_{2} /(v)$ and let $p_{r}: H \rightarrow H$ denote the $r$-radial retraction on $H$, i.e.

$$
p_{r}(x)= \begin{cases}x & \text { if }\|x\| \leq r \\ \frac{r x}{\|x\|} & \text { if }\|x\|>r .\end{cases}
$$

Denote by $F_{1}$ the modification of $F$ given by

$$
F_{1}: T \times H \rightarrow P_{f c}(H), \quad F_{1}(t, x)=F\left(t, p_{r}(x)\right) .
$$

So

- $(t, x) \rightarrow F_{1}(t, x)$ is measurable

- for a.a. $t \in T, x \rightarrow F_{1}(t, x)$ is $h$-upper semicontinuous

- for a.a. $t \in T$, all $x \in H$ and all $v \in F_{1}(t, x),\|v\| \leq c(t)$ with $c \in L^{2}(T)_{+}$.

Now consider the periodic evolution inclusion

$$
\left.\begin{array}{l}
-\dot{x}(t) \in \partial \varphi(x(t))+\left(x(t)-p_{r}(x(t))+F_{1}(t, x(t)) \text { a.e. on } T\right. \\
x(0)=x(b)
\end{array}\right\} .
$$

Suppose we were able to obtain a strong solution $x \in W_{\operatorname{per}(T, H)}^{1,2}$ of it. Then we claim that $\|x\|_{C(T, H)} \leq r$. Suppose that this is not the case. Then $\|x(t)\|>r$ for all $t \in(\beta, \gamma)$ and $\|x(\beta)\|=\|x(\gamma)\|=r$. We know that

$$
-\dot{x}(t)=v(t)+h(t) \quad \text { a.e. on } T
$$


with $v \in S_{\partial \varphi(x(\cdot))}^{2}$ and

$$
h(t)=\left(x(t)-p_{r}(x(t))+f(t) \quad \text { a.e. on } T\right.
$$

where $f \in S_{F_{1}(\cdot, x(\cdot))}^{2}$. Since $0 \in \partial \varphi(0),(v(t), x(t)) \geq 0$ a.e. on $T$. So

$$
(\dot{x}(t), x(t))+(h(t), x(t)) \leq 0 \text { a.e. on } T
$$

and thus

$$
\frac{1}{2} \frac{d}{d t}\|x(t)\|^{2}+(h(t), x(t)) \leq 0 \text { a.e. on } T .
$$

Using (19) we see that, for almost all $t \in[\beta, \gamma]$,

$$
\begin{array}{ll} 
& (h(t), x(t))=\|x(t)\|^{2}-r\|x(t)\|+\frac{\|x(t)\|}{r}\left(f(t), p_{r}(x(t))\right) \\
\Longrightarrow \quad & 0<(h(t), x(t)) \text { a.e. on }[\beta, \gamma] \\
& \left.\quad \text { hypothesis } H(F)_{2} /(v), \text { and recall that } r<\|x(t)\| \text { on }(\beta, \gamma)\right) \\
\Longrightarrow \quad & \frac{1}{2} \frac{d}{d t}\|x(t)\|^{2}<0 \text { a.e. on }(\beta, \gamma) \\
\Longrightarrow & \|x(\gamma)\|^{2}<\|x(\beta)\|^{2} .
\end{array}
$$

The last relation is a contradiction. So every strong solution $x \in W_{p e r}^{1,2}(T, H)$ of problem (18) satisfies $\|x\|_{C(T, H)} \leq r$ and it is obvious that every solution of problem $(18)$ is indeed a solution of problem (1).

Hence, in the sequel we will seek for strong solutions of problem (18). To this end we invoke [13: p. 48/Lemma 3.1] (see also [7]) and we can find a decreasing sequence of multifunctions $F_{1}^{n}: T \times H \rightarrow P_{f c}(H)$ such that:

(a) For all $x \in H, t \rightarrow F_{1}^{n}(t, x)$ is measurable.

(b) For a.e. $t \in T, x \rightarrow F_{1}^{n}(t, x)$ is locally $h$-Lipschitz.

(c) For a.a. $t \in T$, all $x \in H$ and all $v \in F_{1}^{n}(t, x),\|v\| \leq c(t)$.

(d) For a.a. $t \in T$ and all $x \in H, F_{1}^{n}(t, x) \stackrel{h}{\rightarrow} F_{1}(t, x)$ as $n \rightarrow \infty$.

Consider the following approximation to problem (18):

$$
\left.\begin{array}{l}
-\dot{x}(t) \in \partial \varphi(x(t))+\left(x(t)-p_{r}(x(t))+F_{1}^{n}(t, x(t)) \text { a.e. on } T\right. \\
x(0)=x(b)
\end{array}\right\}
$$

Note that for almost all $t \in T$ and all $x \in H$

$$
F_{1}^{n}(t, x) \subset F_{1}(t, x)+2 c(t) \bar{B}_{1} \quad \text { where } \bar{B}_{1}=\{x \in H:\|x\| \leq 1\} .
$$

So if $v \in F_{1}^{n}(t, x)$, then $v=\hat{v}+2 c(t) e$ with $\hat{v} \in F_{1}(t, x)$ and $e \in \bar{B}_{1}$. Now suppose $\|x\| \leq r$. Then

$$
\begin{aligned}
(v, x) & =(\hat{v}+2 c(t) e, x) \\
& \geq(\hat{v}, x)-2 c(t)\|x\| \\
& \geq c_{3}\|x\|^{2}-c_{4}(t)-2 c(t)\|x\| \\
& \geq c_{3}\|x\|^{2}-2 c(t) r-c_{4}(t)
\end{aligned}
$$


provided $(t, x) \in T \times H$ is such that hypothesis $H(F)_{2} /(i v)$ holds. Now if $\|x\|>r$, then

$$
\begin{aligned}
\left(\left(x-p_{r}(x)\right)+v, x\right) & =\|x\|^{2}-r\|x\|+(\hat{v}+2 c(t) e, x) \\
& \geq\|x\|^{2}-r\|x\|-2 c(t)\|x\| \\
& \geq \frac{1}{2}\|x\|^{2}-\frac{1}{2}(r+2 c(t))^{2}
\end{aligned}
$$

where we have used hypothesis $H(F)_{2} /(v)$. From these observations and the fact that $(w, x) \geq 0$ for all $w \in \partial \varphi(x)$ (since $0 \in \partial \varphi(0)$ ) it follows that there exists $\bar{c}_{3}>0$ and $\bar{c}_{4} \in L^{1}(T)_{+}$such that the mapping $(t, x) \mapsto x-p_{r}(x)+F_{1}^{n}(t, x)$ satisfies hypothesis $H(F)_{1} /(i v)$.

We are now in a position to apply Theorem 4 and we obtain a strong solution $x_{n} \in W_{\text {per }}^{1,2}(T, H)$ of problem (20) for every $n \geq 1$. We have

$$
\left.\begin{array}{rl}
-\dot{x}_{n}(t) & =v_{n}(t)+\left(x_{n}(t)+p_{r}\left(x_{n}(t)\right)+f_{n}(t) \text { a.e. on } T\right. \\
x_{n}(0) & =x_{n}(b)
\end{array}\right\}
$$

with $v_{n} \in S_{\partial \varphi\left(x_{n}(\cdot)\right)}^{2}$ and $f_{n} \in S_{F_{1}^{n}\left(\cdot, x_{n}(\cdot)\right)}^{2}$. Taking the inner product with $\dot{x}_{n}(t)$, we obtain

$$
\left\|\dot{x}_{n}(t)\right\|^{2}+\left(v_{n}(t), \dot{x}_{n}(t)\right)+\left(x_{n}(t)+p_{r}\left(x_{n}(t)\right), \dot{x}_{n}(t)\right)+\left(f_{n}(t), \dot{x}_{n}(t)\right)=0
$$

a.e. on $T$. From [13: p. 357] we know that

$$
\left(v_{n}(t), \dot{x}_{n}(t)\right)=\frac{d}{d t} \varphi\left(x_{n}(t)\right) \quad \text { a.e. on } T \text {. }
$$

Therefore

$$
\begin{array}{ll}
\left\|\dot{x}_{n}(t)\right\|^{2}+\frac{d}{d t} \varphi\left(x_{n}(t)\right)+\left(x_{n}(t)+p_{r}\left(x_{n}(t)\right), \dot{x}_{n}(t)\right)+\left(f_{n}(t), \dot{x}_{n}(t)\right)=0 \text { a.e. on } T \\
\Longrightarrow \quad\left\|\dot{x}_{n}\right\|_{2}^{2}=\int_{0}^{b}\left(p_{r}\left(x_{n}(t)\right), \dot{x}_{n}(t)\right) d t-\int_{0}^{b}\left(f_{n}(t), \dot{x}_{n}(t)\right) d t \\
\quad\left(\text { since } x_{n}(0)=x_{n}(b) \text { and } \varphi\left(x_{n}(0)\right)=\varphi\left(x_{n}(b)\right)\right) \\
\Longrightarrow \quad\left\|\dot{x}_{n}\right\|_{2} \leq \sqrt{b} r+\|c\|_{2}=M_{1} .
\end{array}
$$

Then for all $n \geq 1$ and all $s, t \in T$ with $s<t$

$$
\left\|x_{n}(t)-x_{n}(s)\right\| \leq \int_{0}^{t}\left\|\dot{x}_{n}(\tau)\right\| d \tau \leq M_{1} \sqrt{t-s}
$$

from which there follows that $\left\{x_{n}\right\}_{n \geq 1} \subset C(T, H)$ is equicontinuous.

Also, let $\{S(t)\}_{t \in T}$ be the nonlinear semigroup of contractions generated by the maximal monotone operator $\partial \varphi$. From [13: p. 408] we know that, for all $n \geq 1, t \in$ $[0, b)$ and $\lambda>0$ with $t+\lambda \leq b$,

$$
\begin{aligned}
& \left\|J_{\lambda}\left(x_{n}(t)\right)-x_{n}(t)\right\| \\
& \quad \leq \frac{4}{\lambda} \int_{0}^{\lambda}\left\|S(\tau) x_{n}(t)-x_{n}(t)\right\| d \tau \\
& \quad \leq \frac{4}{\lambda} \int_{0}^{\lambda}\left\|S(\tau) x_{n}(t)-x_{n}(t+\tau)\right\| d \tau+\frac{4}{\lambda} \int_{0}^{\lambda}\left\|x_{n}(t+\tau)-x_{n}(t)\right\| d \tau .
\end{aligned}
$$


From [13: p. 408] we also have

$$
\left\|S(\tau) x_{n}(t)-x_{n}(t+\tau)\right\| \leq \int_{t}^{t+\tau} c(s) d s
$$

Therefore exploiting also the equicontinuity of $\left\{x_{n}\right\}_{n \geq 1}$, we see that there exists $0_{t}$, a non-decreasing and continuous function on $T$ such that $0_{t}(0)=0$ and

$$
\left\|J_{\lambda}\left(x_{n}(t)\right)-x_{n}(t)\right\| \leq \frac{4}{\lambda} \lambda 0_{t}(\lambda)=40_{t}(\lambda) \rightarrow 0
$$

from which

$$
\sup _{n \geq 1}\left\|J_{\lambda}\left(x_{n}(t)\right)-x_{n}(t)\right\| \rightarrow 0
$$

follows.

Also, if $t=b$, then

$$
\left\|J_{\lambda}\left(x_{n}(b)\right)-x_{n}(b)\right\|=\left\|J_{\lambda}\left(x_{n}(0)\right)-x_{n}(0)\right\|
$$

and the above argument is still valid. Because $J_{\lambda}$ is compact (since $\varphi$ is of compact type), it follows that, for all $t \in T,\left\{\overline{x_{n}(t)}\right\}_{n \geq 1}$ is compact in $H$. So by the ArzelaAscoli theorem $\left\{x_{n}\right\}_{n \geq 1} \subset C(T, H)$ is relatively compact. Thus we may assume that $x_{n} \rightarrow x$ in $C(T, H)$. Evidently, $x \in W_{\text {per }}^{1,2}(T, H)$ and $\dot{x}_{n} \stackrel{w}{\rightarrow} \dot{x}$ in $L^{2}(T, H)$. Also, there exists $f \in L^{2}(T, H)$ such that $f_{n} \stackrel{\omega}{\rightarrow} f$ in $L^{2}(T, H)$. From [13: p. 694/Proposition vii.3.9] and the properties of the sequence $\left\{F_{1}^{n}\right\}_{n \geq 1}$ we have

$$
f(t) \in \overline{\operatorname{conv}} w-\limsup _{n \rightarrow \infty} F_{1}^{n}\left(t, x_{n}(t)\right) \subset F_{1}(t, x(t)) \text { a.e. on } T
$$

and thus $f \in S_{F_{1}(\cdot, x(\cdot))}^{2}$. Since

$$
-\dot{x}_{n}-\left(x_{n}+p_{r}\left(x_{n}\right)\right)-f_{n} \in \partial \Phi\left(x_{n}\right) \quad(n \geq 1)
$$

we have

$$
-\dot{x}-\left(x+p_{r}(x)\right)-f \in \partial \Phi(x)
$$

and so $x \in W_{\text {per }}^{1,2}(T, H)$ is a strong solution of problem (18). As already observed it follows that $x$ is a strong solution of problem (1). Finally, from the above argument it is clear that the set of solutions of problem (1) is compact in $C(T, H)$ 


\section{Examples}

In this section we present three examples illustrating the applicability of our work.

(a) We start with a nonlinear parabolic variational inequality with discontinuous forcing term. So let $Z \subset \mathbf{R}^{N}$ be a bounded domain with $C^{1}$-boundary $\Gamma$. We consider the parabolic variational inequality

$$
\left.\begin{array}{l}
\frac{\partial x}{\partial t}-\operatorname{div}\left(\|D x(z)\|^{p-2} D x(z)+\beta(x(t, z)) \ni f(t, z, x(t, z))\right. \\
\left.x\right|_{T \times \Gamma}=0, x(0, z)=x(b, z) \text { a.e. on } Z
\end{array}\right\}
$$

where $2 \leq p<\infty$. The right-hand side term $f(t, z, x)$ is discontinuous in $x \in \mathbb{R}$. So following Chang [4], to obtain an existence theorem of problem (21) we pass to a multi-valued forcing term by, roughly speaking, filling in the jumps at the discontinuity points of $f(t, z, \cdot)$. To this end we introduce

$$
\begin{aligned}
& f_{1}(t, z, x)=\liminf _{x^{\prime} \rightarrow x} f\left(t, z, x^{\prime}\right) \\
& f_{2}(t, z, x)=\limsup _{x^{\prime} \rightarrow x} f\left(t, z, x^{\prime}\right) .
\end{aligned}
$$

Then instead of (21) we consider the prob

$$
\left.\begin{array}{c}
\frac{\partial x}{\partial t}-\operatorname{div}\left(\|D x(z)\|^{p-2} D x(z)+\beta(x(t, z))\right. \\
-\left[f_{1}(t, z, x(t, z)), f_{2}(t, z, x(t, z))\right] \ni 0 \\
\left.x\right|_{T \times \Gamma}=0, x(0, z)=x(b, z) \text { a.e. on } Z
\end{array}\right\}
$$

with $2 \leq p<\infty$. We solve this new problem. The hypotheses of the data are the following ones:

$\mathbf{H}(\beta)_{1} \quad \beta: \mathbb{R} \rightarrow 2^{\mathbb{R}}$ is a maximal monotone map with $0 \in \beta(0)$ (hence $\beta=\partial j$ with $\left.j \in \Gamma_{0}(\mathbb{R})\right)$.

$\mathbf{H}(\mathbf{f})_{1} f: T \times Z \times \mathbb{R} \rightarrow \mathbb{R}$ is a Borel measurable function such that $|f(t, z, x)| \leq$ $c_{1}(t, z)+c_{2}(t, z)|x|$ a.e. on $T \times Z$ with $c_{1}, c_{2} \in L^{2}(T \times Z)_{+}, f_{1}, f_{2}$ are both jointly measurable and, for almost all $(t, z) \in T \times Z$ and all $x \in \mathbb{R}$, $f(t, z, x) x \geq c_{3}(z)|x|^{2}-c_{4}(t, z)$ with $c_{3} \in L^{\infty}(Z)$ and $c_{4} \in L^{\infty}(T \times Z)$.

Let $H=L^{2}(Z)$ and

$$
\varphi(x)= \begin{cases}\frac{1}{p}\|D x\|_{p}^{p}+\int_{Z} j(x(z)) d z & \text { if } x \in W^{1,2}\left(Z, j(x(\cdot)) \in L^{1}(Z)\right. \\ +\infty & \text { otherwise. }\end{cases}
$$

Evidently, $\varphi \in \Gamma_{0}(H)$ (see [20: p. 194]) and $\partial \varphi(x)=-\operatorname{div}\left(\|D x\|^{p-2} D x\right)+S_{\beta(x(\cdot))}^{2}$ (see [20: p. 195]). Note that $0 \in \partial \varphi(0)$ and by virtue of the Sobolev embedding theorem it is of compact type. Also, set

$$
-F(t, x)=\left\{-h \in H: f_{1}(t, z, x(z)) \leq h(z) \leq f_{2}(t, z, x(z)) \text { a.e. on } Z\right\} .
$$

Using hypotheses $H(f)_{2}$, we can easily check that hypothesis $H(F)_{2}$ holds. Now rewrite (22) in the equivalent abstract evolution inclusion form (1) and apply Theorem 5 to deduce 
Proposition 6. If hypotheses $H(\beta)_{1}$ and $H(f)_{1}$ hold, then problem (22) has a solution $x \in C\left(T, L^{2}(Z)\right)$ such that $\frac{\partial x}{\partial t} \in L^{2}(T \times Z)$.

(b) We consider a semilinear parabolic control system with a priori feedback and non-homogeneous, multi-valued Neumann boundary conditions. So $Z \subset \mathbb{R}^{N}$ is as before and, for $x \in W^{1,2}\left(Z, \mathbb{R}^{N}\right), L x=\left(\Delta x_{k}\right)_{k=1}^{N}$ with $\hat{x}=\left(x_{k}\right)_{k=1}^{N}$. We consider the problem

$$
\left.\begin{array}{l}
\frac{\partial x}{\partial t}-L x(t, z)=f(t, z, x(t, z), u(t, z)) \\
\left.x\right|_{T \times \Gamma}=0, x(0, z)=x(b, z) \text { a.e. on } Z \\
u(t, z) \in U(t, z, x(t, z)) \text { a.e. on } T \times Z
\end{array}\right\} .
$$

The hypotheses on the data are the following ones:

$\mathbf{H}(\beta)_{2} \quad \beta=\partial j$ with $j \in \Gamma_{0}\left(\mathbb{R}^{N}\right)$ and $j(0)=\inf _{\mathbb{R}^{N}} j \geq 0$.

$\mathbf{H ( f )})_{2} f: T \times Z \times \mathbb{R}^{N} \times \mathbb{R}^{m} \rightarrow \mathbb{R}^{N}$ is a function such that the following conditions are satisfied:

(i) For all $(x, u) \in \mathbb{R} \times \mathbb{R}^{m},(t, z) \rightarrow f(t, z, x, u)$ is measurable.

(ii) For all $(t, z) \in T \times Z,(x, u) \rightarrow f(t, z, x, u)$ is continuous.

(iii) For all $r>0$ there exist $c_{1 r}, c_{2 r} \in L^{2}(T \times Z)$ such that $\|f(t, z, x, u)\| \leq$ $c_{1 r}(t, z)+c_{2 r}(t, z)\|x\|$ for a.a. $(t, z) \in T \times Z$, all $x \in \mathbf{R}$ and all $\|u\| \leq r$.

(iv) For a.a. $(t, z) \in T \times Z$, all $x \in \mathbf{R}^{N}$ and all $u \in \mathbb{R}^{m}$ with $\|u\| \leq r$, $(f(t, z, x, u), x)_{\mathbb{R}^{N}} \geq c_{3}\|x\|^{2}-c_{4}(t, z)$ with $c_{3}>0$ and $c_{4} \in L^{1}(T \times Z)$.

$\mathbf{H}(\mathrm{U}) U: T \times Z \times \mathbb{R}^{N} \rightarrow P_{k}\left(\mathbb{R}^{m}\right)$ is measurable, for almost all $(t, z) \in T \times Z$, $U(t, z, \cdot)$ is lower semicontinuous and, for a.a. $(t, z) \in T \times Z$, all $x \in \mathbf{R}^{N}$ and all $u \in U(t, z, x),\|u\| \leq M$.

Let $H=L^{2}\left(Z, \mathbb{R}^{N}\right)$ and let $\varphi: H \rightarrow \overline{\mathbb{R}}=\mathbb{R} \cup\{+\infty\}$ be defined by

$$
\varphi(x)= \begin{cases}\frac{1}{p}\|D x\|_{2}^{2}+\int_{\Gamma} j(x(z)) d \sigma & \text { if } x \in W^{1,2}\left(Z, \mathbb{R}^{N}\right), j(x(\cdot)) \in L^{1}(\Gamma) \\ +\infty & \text { otherwise. }\end{cases}
$$

From [3: p. 63] we know that $\varphi \in \Gamma_{0}(H)$ and $\partial \varphi(x)=-L x$ with domain

$$
D(\partial \varphi)=\left\{x \in W^{2,2}\left(Z, \mathbb{R}^{N}\right):-\frac{\partial x}{\partial n} \in \beta(x(z)) \text { a.e. on } \Gamma\right\} .
$$

Set $\hat{f}(t, z, x)=f(t, z, x, U(t, z, x))$ and $F(t, x)=S_{\hat{f}(t, ;, x(\cdot))}^{2}$ for all $(t, x) \in T \times H$. Using hypotheses $H(f)_{2}$ and $H(U)$ it is routine to check that hypotheses $H(F)_{1}$ hold. So we can apply Theorem 4 and deduce

Proposition 7. If hypotheses $H(\beta)_{2}, H(f)_{2}$ and $H(U)$ hold, then problem (23) has a solution $x \in C\left(T, L^{2}\left(Z, \mathbf{R}^{N}\right)\right)$ with $\frac{\partial x}{\partial t} \in L^{2}\left(T \times Z, \mathbf{R}^{N}\right)$.

(c) Our formulation also incorporates differential variational inclusions

$$
\left.\begin{array}{l}
-\dot{x}(t) \in N_{K}(x(t))+F(t, x(t)) \text { a.e. on } T \\
x(0)=x(b)
\end{array}\right\}
$$


Herein $N_{K}(x)$ is the normal cone to $K$ at $x \in K$, with $K \in P_{f c}\left(\mathbf{R}^{N}\right)$. Problems like (24) arise in theoretical mechanics and economics (see [14]). In fact, (24) is equivalent to the projected system

$$
\left.\begin{array}{l}
-\dot{x}(t) \in \operatorname{proj}\left(-F(t, x(t)) ; T_{K}(x(t))\right) \text { a.e. on } T \\
x(0)=x(b)
\end{array}\right\}
$$

Here $T_{K}(x)$ is the tangent cone to $K$ at $x$. We know $N_{K}(x)^{-}=T_{K}(x)$. Inclusions like (25) arise in the study of systems with constraints (see, for example, [15]). The hypotheses on $F(t, x)$ are:

$\mathbf{H}(\mathbf{F})_{3} \quad F: T \times \mathbb{R}^{N} \rightarrow P_{k}\left(\mathbb{R}^{N}\right)$ is a multifunction such that the following conditions are satisfied:

(i) $(t, x) \rightarrow F(t, x)$ is graph measurable.

(ii) For a.a. $t \in T, x \rightarrow F(t, x)$ is lower semicontinuous.

(iii) For a.a. $t \in T$, all $x \in \mathbb{R}^{N}$ and all $v \in F(t, x),\|v\| \leq c_{1}(t)+c_{2}(t)\|x\|$ with $c_{1}, c_{2} \in L^{2}(T)_{+}$.

(iv) For a.a. $t \in T$, all $x \in \mathbb{R}^{N}$, all $w \in N_{K}(x)$ and all $v \in F(t, x),(w+v, x)_{\mathbb{R}^{N}} \geq$ $c_{3}\|x\|^{2}-c_{4}(t)$ with $c_{3}>0$ and $c_{4} \in L^{1}(T)_{+}$.

Using Theorem 4 with

$$
\varphi(x)=\delta_{K}(x)= \begin{cases}0 & \text { if } x \in K \\ +\infty & \text { otherwise }\end{cases}
$$

we obtain

Proposition 8. If hypotheses $H(F)_{3}$ hold and $K \subset \mathbf{R}^{N}$ is non-empty, closed convex with $0 \in K$, then problem (24) (equivalently problem (25)) has a solution $x \in W^{1,2}\left(T, \mathbf{R}^{N}\right)$.

In particular, if $K=\left\{x \in \mathbb{R}^{N}: 0 \leq x \leq \xi\right\}$ with $\xi \in \mathbf{R}_{+}^{N}$, we obtain

$$
\begin{aligned}
& -\dot{x}(t) \in F(t, x(t)) \text { a.e. on }\{t \in T: 0<x(t)<\xi\} \\
& -\dot{x}(t) \in F(t, x(t))-\mathbb{R}_{+}^{N} \text { a.e. on }\{t \in T: x(t)=0\} \\
& -\dot{x}(t) \in F(t, x(t))+\mathbb{R}_{+}^{N} \text { a.e. on }\{t \in T: x(t)=b\} \\
& x \in W^{1,2}\left(T, \mathbb{R}^{N}\right), x(0)=x(b) \\
& 0 \leq x(t) \leq \xi \text { for all } t \in T
\end{aligned}
$$




\section{References}

[1] Avgerinos, E. and N. S. Papageorgiou: Solutions and periodic solutions for nonlinear evolution equations with non-monotone perturbations. Z. Anal. Anw. 17 (1998), 859 875.

[2] Bader, R.: On the semilinear multivalued flow under constraints and the periodic problem. Comment. Math. Univ. Carolinae 41 (2000), 719 - 734.

[3] Barbu, V.: Nonlinear Semigroups and Differential Equations in Banach Spaces. Leyden: Noordhoff Int. Publ. 1976.

[4] Chang, K.-C.: Variational methods for nondifferentiable functionals and its applications to partial differential equations. J. Math. Anal. Appl. 80 (1981), $102-129$.

[5] Christensen, J. P. R.: Topology and Borel Structure. Amsterdam: North Holland 1981.

[6] De Blasi, F., Gorniewicz, L. and G. Pianigiani: Topological degree and periodic solutions of differential inclusions. Nonlin. Anal. 37 (1999), 217 - 245.

[7] De Blasi, F. and G. Pianigiani: Hausdorff measurable inultifunctions. J. Math. Anal. Appl. 228 (1998), 1 - 15.

[8] Haddad, G. and J.-M. Lasry: Periodic solutions of functional differential inclusions and fixed points of $\sigma$-selectionable correspondences. J. Math. Anal. Appl. 96 (1983), $295-312$.

[9] Hirano, N.: Existence of periodic solutions for nonlinear evolution equations in Hilbert spaces. Proc Amer. Math. Soc. 120 (1994), 185 - 192.

[10] $\mathrm{Hu}$, S., Kandilakis, D. and N. S. Papageorgiou: Periodic solutions for nonconvex differential inclusions. Proc. Amer. Math. Soc. 127 (1999), 89 - 94.

[11] Hu, S. and N. S. Papageorgiou: On the existence of periodic solutions for nonconvex differential inclusions in $\mathbb{R}^{\mathbf{N}}$. Proc. Amer. Math. Soc. 123 (1995), 3043-305.

[12] $\mathrm{Hu}, \mathrm{S}$. and N. S. Papageorgiou: On the existence of periodic solutions for a class of nonlinear evolution inclusions. Bollettino UMI 7-B (1993), $591-605$.

[13] Hu, S. and N. S. Papageorgiou: Handbook of Multivalued Analysis. Volume I: Theory. Dordrecht: Kluwer 1997.

[14] Hu, S. and N. S. Papageorgiou: Handbook of Multivalued Analysis. Volume II: Applications. Dordrecht: Kluwer 2000.

[15] Krasnoselskii, M. and A. Pokrovskii: Systems with Hysteresis. Berlin: Springer-Verlag 1988.

[16] Macki, J., Nistri, P. and P. Zecca: The existence of periodic solutions for nonautonomous differential inclusions. Proc. Amer. Math. Soc. 104 (1988), $840-844$.

[17] Marcus, M. and V. Mizel: Absolute continuity on tracks and mappings of Sobolev spaces. Arch. Rat. Mech. Anal. 45 (1972), 294 - 320.

[18] Prüss, J.: Periodic solutions of semilinear evolution equations. Nonlin. Anal. 3 (1979), $601-612$.

[19] Pruszko, T.: Some applications of the topological degree theory to multivalued boundary value problems (Dissertationes Math.: Vol. 129). Warsaw: Polish Acad. Sci. 1984.

[20] Showalter, R.: Monotone Operators in Banach Space and Nonlinear Partial Differential Equations (Math. Surveys: Vol 49). Providence, R.I. (USA): Amer. Math. Soc. 1998.

[21] Vrabie, I.: Periodic solutions for nonlinear evolutions equations in a Banach space. Proc. Amer. Math. Soc. 109 (1990), 653 - 661. 
984

R. Bader and N. S. Papageorgiou

[22] Zeidler, E.: Nonlinear Functional Analysis and its Applications. Vol. II A/B: Nonlinear/Linear Monotone Operators. New York: Springer-Verlag 1990.

Received 18.03.2002 